\title{
Pain: lessons from the past can inform the present and future
}

\section{Michelle Witkop}

Pain is a phenomenon that accompanies a person with haemophilia (PWH) and many others with bleeding disorders from birth to death. Caregivers are not immune. For you cannot provide care, either as a loved one or a health care provider, and watch someone in pain without experiencing pain yourself.

In many ways, the elders of the haemophilia community in developed countries are pioneers. Theirs is a story to be told, much different than those in undeveloped countries. Pain often directs that story. Their families and children are living the consequences of these pioneer's lives. They are experiencing many "firsts": the first to live beyond their 30s, the first to live through the HIV crisis, the first to require screening procedures for ageing (colonoscopies, etc.) and to be afflicted with diseases of ageing, and the first to live with chronic pain into their advanced years. They are experiencing these "firsts" because their life span is now approaching the lifespan of the general population as a consequence of improvements in medical care [1]. The experiences of these elders is captured in three articles in this issue of The Journal of Haemophilia Practice.

In the article by Pollard and Moore [2], eight men describe their haemophilia journeys and its effect on their lives. Several of these men would now be considered among those "elders". Despite the pain and psychosocial consequences of haemophilia that affect not only the patient but also his family, these men are living what they describe as a good quality of life.

Our younger PWH are watching and learning from these pioneers as are their healthcare providers. We are learning how they have coped, how they live with daily pain and continue to function (and in certain cases, how they do not function because of the pain), and how they are resilient. For those who have developed that resilience, the question is how? How can it be replicated? Can it be encouraged or taught and if so, how? These are questions that stimulate further research, particularly, qualitative nursing research. For those who still do not have the benefit of readily available clotting factor, either for prophylaxis or to easily treat ondemand (such as the mild haemophilia patients described by Alavian et al [3]), the questions are similar. What is their

Michelle Witkop DNP, FNP-BC, Doctor of Nursing Practice and Bleeding Disorders Nurse Practitioner, Munson Medical Center, Traverse City, Michigan, USA. Email: mwitkopamhc.net

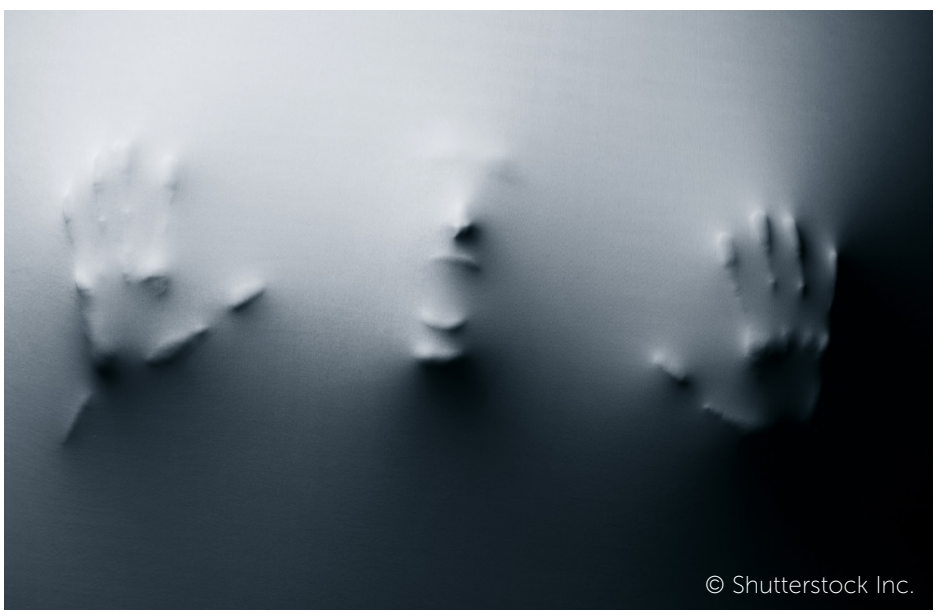

pain experience? How do they and their caregivers cope and live with pain that affects their daily lives? How do they maintain function?

The consequences of unresolved/untreated pain are high. Not only for the experiencing person but also for those who love and care for that person. Many mothers can describe their physical reaction when they think of their son experiencing a joint bleed. Along with the $\mathrm{PWH}$, many parents and family members go on to experience posttraumatic stress disorder (PTSD) that impacts their lives for years to come. PTSD affects how they interact with the healthcare system, how they respond to pain themselves, and the goals/expectations they develop for the future. As described in several of the accompanying articles, watching others live in pain as well as living in pain yourself can even affect family planning decisions for the future.

The articles by Rambod et al [4] and Pollard and Moore [2] both demonstrate these phenomena. The consequences of unresolved/untreated pain are detailed in the descriptions of pain by the participants. Continued research to document this experience is important not only to validate the patient's perspective but also to allow the healthcare provider a glimpse into that experience thus increasing the opportunity that future pain will be treated appropriately.

The way that providersassess and treat pain is equally important. While it is important to validate a person's pain, it is also important not to feed into catastrophising that pain or suggesting addiction when no symptoms of that disease are present. The words we use, the language we chose, and how we share that with others is of utmost significance 
as we move forward in our struggle to recognise and treat pain as the disease entity it is and not to assign it to a psychological fault. While many $\mathrm{PWH}$ require opioids to manage their pain, they experience the stigma associated with their use $[5,6]$.

Equipped with this knowledge, it is imperative that the haemophilia community takes action to treat and prevent pain in those with haemophilia and other bleeding disorders. First and foremost, when available, prevention is key. The use of clotting factor as primary or secondary prophylaxis to prevent bleeds is vital to preventing pain in this population. Once haemarthrosis occurs, the next step is the proper assessment of pain followed by a treatment plan that is multimodal in nature [7].

As providers we must recognise that a purely pharmaceutical approach is not only out of reach for a majority of the global haemophilia population, it is also inappropriate and an unproven method of pain management. In developed countries, opioid usage is under attack on many fronts while in developing countries opioids are just not available. $\mathrm{PWH}$ who live with pain in developed countries, even those with mild disease as presented by Alavian et al [3], may learn strategies from $\mathrm{PWH}$ who live with pain in countries in which haemophilia care is still evolving as presented by Rambod et al $[3,4]$. In all three articles, both are resilient but employed different methods of dealing with the pain they have to live with daily. Nurse-led research studies, such as those published in The Journal of Hemophilia Practice, offer a greater understanding of how $\mathrm{PWH}$ manage pain as well as other aspects of their lives. They are a valuable addition on the road of sharing information and learning from each other.

\section{Disclosures}

The author has advised that there are no interests that might be perceived as posing a conflict or bias.

This is an Open Access article distributed under the terms of the Creative Commons Attribution License (http://creativecommons.org/licenses/ by/2.0), which permits unrestricted use, distribution, and reproduction in any medium, provided the original work is properly cited.

\section{References}

1. Evatt BL. The natural evolution of haemophilia care: developing and sustaining comprehensive care globally. Haemophilia 2006; 12: 13-21. doi: 10.1111/j.1365-2516.2006.01256.x

2. Pollard D, Moore J. What is the impact on quality of life of living with severe haemophilia. J Haem Pract 2016; 3(1). doi: 10.17225/jhp00068

3. Alavian S, Norton C, Othman S. Living with mild haemophilia and care following a bleed - a qualitative study. J Haem Pract 2016; 3(1). doi: 10.17225/jhp00063

4. Rambod M, Sharif F, Molazem Z, Khair K. Pain: the voiceless scream in every haemophilia patient's life. J Haem Pract 2016; 3(1). doi: 10.17225/ jhp00061

5. Broyles LM, Binswanger IA, Jenkins JA, Finnel DS, Faseru B, Cavaiola A, Pugatch M, Gordon AJ. Confronting inadvertent stigma and pejorative language in addiction scholarship: a recognition and response. Substance Abuse 2014; 35:3: 217-221. doi: 10.1080/08897077.2014.930372

6. Monsivais, D. Decreasing the stigma burden of chronic pain. Journal of American Association of Nurse Practitioners 2013; 25: 551-556.

7. Riley RR, Witkop M, Hellman E, Akins S. Assessment and management of pain in haemophilia patients. Haemophilia 2011; 17(6): 839-45. doi: 10.1111/j.1365-2516.2011.02567.x.2516.2011.02567.x.

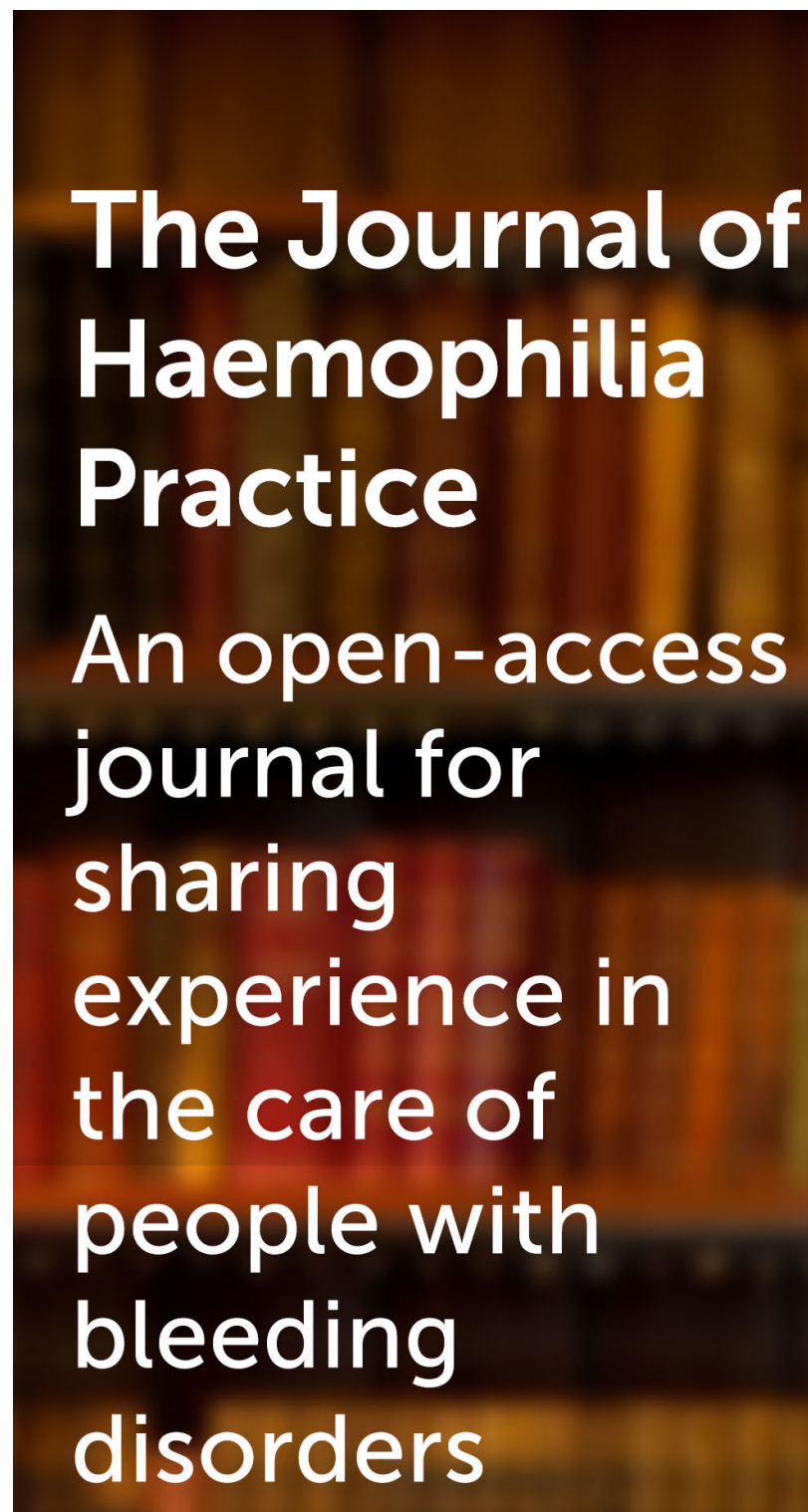

\section{www.haemjournal.com}

